\title{
W-visible submodules and W-fully visible modules
}

\author{
Mahmood S. Fiadh ${ }^{1 *}$, Buthyna N. Shihab², Munther H. Abed ${ }^{3}$ \\ ${ }^{1}$ Dep. of computer Sci./ College of Edu./ AlIraqia Univer./ Baghdad / Iraq. \\ ${ }^{2}$ Dep. of Math./ College of Edu. for Pure Sciences Ibn AL-Haitham /Univ. of Baghdad/ Baghdad / Iraq. \\ ${ }^{3}$ Faculty of Computing, College of Computing and Applied Sciences, Universiti Malaysia Pahang, Malaysi
}

*Corresponding Author: Mahmood S. Fiadh

DOI: https://doi.org/10.52866/ijcsm.2019.01.01.005

Received December 2019; Accepted December 2019; Available online January 2020

ABSTRACT: In this paper, we presented a special type of submodule named W-visible submodule, which is weaker than the visible submodule, where a proper submodule $\mathrm{W}$ of a T-module $\mathrm{X}$ is said to be $\mathrm{W}$-visible if $\mathrm{W}=\mathrm{IW}$ for some non-zero proper ideal I of $\mathrm{T}$. Also, we presented the concept of $\mathrm{W}$-fully visible module, where a module $\mathrm{X}$ over a ring $\mathrm{T}$ is said to be $\mathrm{W}$-fully visible if any proper submodule of $\mathrm{X}$ is $\mathrm{W}$-visible submodule. This concept is considered weaker than the concept of fully visible module. In this study, we achieved numerous results and characteristics that belong to this concept.

Keywords: Visible submodule; W-visible submodule; Fully visible module; W-fully visible module.

\section{INTRODUCTION}

Let $X$ be a unitary module and $T$ be a commutative ring. In [1], Mahmood and Buthyna submitted a concept of visible submodule which is defined as a proper submodule $W$ of a module $X$ over a ring $T$ to achieve $W=I W$ for every non-zero ideal $I$ of $T$. Also, Mahmood [2] presented the concept of fully visible module, which is defined as the module in which all submodules are visible.

In section two of this paper, a special type of submodule that is weaker than the visible submodule was presented; this submodule has been called the $W$-visible submodule. In this submodule, a proper submodule $W$ of a $T$-module $X$ is said to be $W$-visible if $W=I W$ for some non-zero proper ideal $I$ of $T$.

It is clear that every visible submodule is $W$-visible, but the converse is not true, and we have been able to give an example of this.

In section three, we presented the concept of $W$-fully visible module, which is a module filled with $W$-visible submodules; in other words, a module $X$ over a ring $T$ is said to be $W$-fully visible if any proper submodule of $X$ is $W$-visible submodule. This concept is considered weaker than the concept of fully visible module.

Many characterizations of $W$-visible submodules and $W$-fully visible modules have been submitted to many different properties. 


\section{W-VISIBLE SUBMODULES AND SOME OF THE PROPERTIES}

Here, we introduce the concept of $W$-visible submodules which is considered weaker than the concept of visible submodules. Several results have been proven and the advantages have been given to describe this definition.

Definition 2.1. A proper submodule $W$ of an $T$-module $X$ is called $W$-visible if there exists a non-zero proper ideal $C$ of $T$, such that $W=C W$.

\section{Remarks and Examples 2.1.}

1) A zero submodule of some $T$-module $X$ can be $W$-visible, but in others, a zero submodule can be non- $W$-visible. In particular, $Z_{P}$ as a $Z_{P}$-module, $P$ is a prime number and $Q$ as a $Q$-module has a zero submodule and cannot be W-visible.

2) A submodule $(\overline{2})$ of a $Z$-module $Z_{4}$ is $W$-visible since there exists a non-zero proper ideal $C=(3)$ of $Z$ such that $(\overline{2})=(3)(\overline{2})$. Also, the submodules $(\overline{2})$ and $(\overline{3})$ in a module $Z_{6}$ on a ring $Z$ are $W$-visible since $(\overline{2})=$ (2) $(\overline{2})$ and $(\overline{3})=(3)(\overline{3})$ for some ideals (2) and (3) of Z, respectively.

3) In a more general form, any proper submodule of a $Z$-module $Z_{n}, n$, is an integer and can be $W$-visible.

4) All proper submodules, except the zero submodule of a module $Z_{n}$ on a ring $Z_{n}$, $n$, are an integer and not prime; $n>1$ cannot be $W$-visible.

5) In a module $Z_{12}$ as $Z_{36}$-module, there are proper submodules represented by $(\overline{3}),(\overline{4})$ and $(\overline{6})$ be $W$-visible. Since there are $(\overline{9})$ and $(\overline{4})$, they can be ideals of $Z_{36}$ such that $(\overline{3})=(\overline{9})(\overline{3}),(\overline{4})=(\overline{4})(\overline{4})$ and $(\overline{6})=$ $(\overline{9})(\overline{6})$. At the same module $Z_{12}$, there is an ideal $(\overline{2})$ that could not be $W$-visible since $(\overline{2}),(\overline{4}),(\overline{6}),(\overline{9})$ and

(12) are non-zero proper ideals of $Z_{36}$. But,

$$
\begin{aligned}
& (\overline{2})(\overline{2})=(\overline{4}) \neq(\overline{2}) \\
& (\overline{4})(\overline{2})=(\overline{8}) \neq(\overline{2}) \\
& (\overline{6})(\overline{2})=(\overline{0}) \neq(\overline{2}) \\
& (\overline{9})(\overline{2})=(\overline{6}) \neq(\overline{2}) \\
& (\overline{12})(\overline{2})=(\overline{0}) \neq(\overline{2})
\end{aligned}
$$

That is, $(\overline{2})$ is not a $W$-visible submodule of $Z_{12}$.

6) The submodules $(\overline{2})$ and $(\overline{4})$ are not $W$-visible in a module $Z_{8}$ over a ring $Z_{16}$, because there is no ideal $C$ of $Z_{16}$ such that $(\overline{2})=C(\overline{2})$ and $(\overline{4})=C(\overline{2})$.

7) Every non-zero submodule $W$ of a $Z$-module $Z$ is not $W$-visible, since there is no non-zero proper ideal $C$ of a ring $Z$ such that $W=C W$.

8) Consider the module $Z \oplus Z_{n}$ as a $Z$-module; $n$ is an integer, $n>1$, all the proper submdoules of $Z \oplus Z_{n}$ which are in the form of $(0) \oplus W$ and $W$ are a submodule of $Z_{n}$ and can be $W$-visible. Other proper submodules of $\cap \oplus Z_{n}$ that are in the form $W_{1} \oplus W_{2}$, where $W_{1}$ is a non-zero submodule of $Z$ and $W_{2}$ is any submodule of $Z_{n}$, cannot be $W$-visible. 
9) As for (8), if $n=1$, then, all non-zero proper submodules of a module $Z \oplus Z$ over $Z$ are not $W$-visible.

10) A visible submodule of $X$ is always $W$-visible submodule, but the converse is not true in general, and the example below shows that:

The submodule $(\overline{2})$ of a $Z$-module $Z_{4}$ is $W$-visible by (2), but it is not visible because we cannot guarantee that $(\overline{2})=C(\overline{2})$ for all non-zero proper ideals of a ring $Z$.

11) Any proper submodule of W-visible submodule is also W-visible

Proof. (11):

Let $K$ be a proper submodule of $W$-visible $W$; then, there exists a non-zero proper ideal $C$ of $T$ such that $W=C W$, $K \subseteq W=C W$, implying that $K+C W=C W \ldots(1)$

Also, from the same above inclusion, we get:

$C K \subseteq C W$, implying that $C W+C K=C W \ldots(2)$

From (1) and (2), we get $C W+K=C W+C K$ and hence, $K=C K$.

Thus, $K$ is $a W$-visible submodule of $W$.

The opposite of (11) is not always true; let us illustrate this with the example below:

$(0)$ is a $W$-visible submodule of a $Z$-module $Z$ by (1), and $(0) \subseteq(2)$, where (2) is a submodule of the module $Z$ over a $\operatorname{ring} Z$, but $(2)$ is not $W$-visible by $(7)$.

In number (12), we discuss the intersection of two $W$-visible submodules.

12) Let $W_{1}$ and $W_{2}$ be two submodules of $X$ which at least one of them is $W$-visible $T$-submodule. Then, $W_{1} \cap W_{2}$ is also $W$-visible $T$-submodule.

Proof. (12):

It is known that $W_{1} \cap W_{2} \subseteq W$; also, $W_{1} \cap W_{2} \subseteq W_{2}$. Then, by (11), we obtain $W_{1} \cap W_{2}$ which is a $W$-visible submodule. As a generalization of (12), we get:

13) If $\left(W_{i}\right\}_{i=1}^{n}$ is a finite collection of submodules and $W_{i}$ is a $W$-visible submodule for some $i$, then, $\bigcap_{i=1}^{n} W_{i}$ is also $W$-visible.

\section{Proof. (13):}

By induction on $n$;

14) Let $T$ be a field; then, every proper submodule of a $T$-module $X$ is not $W$-visible.

\section{Proof. (14):}

Suppose $K$ is a proper submodule of $X$; since $T$ is a field, then, $T$ has only two ideals $(0)$ and $T$, and hence, we can not find a non-zero ideal $I$ of $T$ such that $K=I K$. Therefore, $K$ is not a $W$-visible submodule of $X$.

Proposition 2.1. Let $X$ and $N$ be two $T$-modules and $f: X \longrightarrow N$ be a T-epimorphism.

1) If $W$ is a $W$-visible submodule of $X$, then, $f(W)$ is a $W$-visible submodule of $N$.

2) If $K$ is a $W$-visible submodule of $N$, then, $f^{-1}(K)$ is a $W$-visible submodule of $X$.

\section{Proof. (1)}

Let $W$ be a proper $W$-visible submodule of $X$. Then, $W=I W$ for some non-zero proper ideal $I$ of $T \cdot f(W)=f(I W)=$ If $(W)$. Therefore, $f(W)$ is a $W$-visible submodule of $N$.

The condition $f$ is an epimorphism and is not necessary here since we can take $f: Z_{4} \longrightarrow Z_{4}$ such that $f(x)=4 x \forall x \in Z_{4}$ , where $Z_{4}$ is a $Z$-module. It is easy to show that $f$ is not epimorphic. Let,$- W$ is a $W$-visible submodule of $Z_{4}$ and - is also a $W$-visible of $Z_{4}$.

Proof. (2)

Let $K$ be a $W$-visible submodule of $N$, then, there exists a non-zero proper ideal $J$ of $T$ such that $K=J K$; therefore, $f^{-1}(K)=f^{-1}(J K)=J f^{-1}(K)$ and hence, $f^{-1}(K)$ is a $W$-visible submodule of $X$.

In the following, we are working characterizations for the $W$-visible submodule.

Proposition 2.2. Let $X$ be a $T$-module and $N$ be a proper submodule of $X$. Then, the following are equipollent:

1) $N$ is $W$-visible. 
2) $(I N: N)=T$ for some nonzero proper ideal I of $T$.

3) $(r N: N)=T$ for nonzero element $r \in T, r \neq 1$.

4) $N=r N$ for some nonzero element $r \in T, r \neq 1$.

Proof.

$(1) \Longrightarrow(2)$ Suppose that $N$ is $W$-visible, then, $I N=N$ for some non-zero proper ideal $I$ of $T$ and hence, $(I N: N)=$ $(N: N)=T$. Therefore, (2) holds.

(2) $\Longrightarrow(3)$ The proof is direct.

(3) $\Longrightarrow$ (4) From (3), we get $N \subseteq r N$ for some non-zero $r \in T, r \neq 1$, also, we have $r N \subseteq N$. Therefore, $r N=N$.

(4) $\Longrightarrow(1)$ Suppose (4) holds, then, $N=(r) N$ for some non-zero $r \in T, r \neq 1$. Therefore, take $I=(r)$ and hence, $N=I N$ which implies $N$ is a $W$-visible submodule of $X$.

Proposition 2.3. Let $X_{1}$ and $X_{2}$ be two $T$-modules and $X=X_{1}+X_{2}$. Let $W$ be a proper submodule of $X$. If $W=K+L$ for some $K \leq X_{1}, L \leq X_{2}$ such that $W$ is $W$-visible submodule. Then, each of $K$ and $L$ are $W$-visible submodules.

Proof. We have $W$ as a $W$-visible submodule of $X$, then, $I W=W$ for some non-zero ideal $I$ of $T$, but $W=K+L$, then, $I(K+L)=K+L$ and hence, $I K+I L=K+L$. Therefore, $I K=K$ and $I L=L$ which implies $L, K$ are $W$-visible submodules of $X_{1}, X_{2}$, respectively.

Remark 2.1. The opposite of Proposition (2.5) does not hold in general; since the Z-module $X=Z_{10}+Z_{9}$. If $W=$ $(\overline{5})+(\overline{3})$, where $(\overline{5})$ and $(\overline{3})$ are $W$-visible subnmodules of $Z_{10}$ and $Z_{9}$, respectively by Remarks and Examples ((2.2),(2)), but $W$ is not a $W$-visible submodule.

Next, we explain the behavior of $W$-visible submodules of a module $X$ over $T$ under localization.

Proposition 2.4. Let $X$ be a T-module; then, a proper submodule $N$ of $X$ is $W$-visible if and only if $N_{P}$ is $W$-visible submodule of a $T_{P}$-module $X_{P}$ for every maximal ideal $P$ of $T$.

Proof. First direction: Let $N$ be a proper submodule of $X$. To prove that $N$ is a W-visible submodule of $X$; since $N_{P}$ is a $W$-visible submodule of $X_{P}$, then, there exists a non-zero proper ideal $I_{P}$ of $T_{P}$ such that $N_{P}=I_{P} N_{P}$ which implies $N_{P}=(I N)_{P}$ for every maximal ideal $P$ of $T$ by [4] and hence, $N=I N$ for some non-zero proper ideals $I$ of $T$. Therefore, $N$ is a $W$-visible submodule of $X$.

Second direction: Suppose $N$ is a $W$-visible submodule of $X$ over $T$; To prove $N_{P}$ is a $W$-visible submodule of $X_{P}$ over $T_{P}$ (for every maximal ideal $P$ of $T$ ), $N=I N$ for some non-zero proper ideals $I$ of $T$ which implies $N_{P}=(I N)_{P}$ (for every maximal ideal $P$ of $T)$. And by [4], we get $N_{P}=I_{P} N_{P}$ since $I_{P}$ is a non-zero proper ideal of $T_{P}$. This leads to the proof that $N_{P}$ is a $W$-visible submodule of $X_{P}$

\section{W-FULLY VISIBLE MODULES}

In this section, the concept of $W$-fully visible has been introduced, where the concept of the fully visible module is stronger than the concept of $W$-fully visible and we were able to give an example in this regard.

Some characterizations and many properties of this concept are proven; also, the tensor product of two $W$-fully visible modules is studied by giving some important results that illustrate it.

In the following, we submit the concept of $W$-Fully visible modules.

Definition 3.1. Let $X$ be a $T$-module; then, $X$ is called $W$-fully visible if every proper submodule of $X$ is $W$-visible.

Remarks and Examples 3.1.

1) Consider the module $Z_{n}$ over a ring $Z$; $n$ is a positive integer and $a W$-fully visible module.

2) $Z_{n}$ as a $Z_{n}$-module, $n$ is a positive integer and $n \geq 1$ is not $W$-fully visible.

3) The module $Z \oplus Z_{n}$ over $Z$, $n$ is a positive integer and $n \geq 1$ is not a $W$-fully visible module.

4) $Z_{P^{\infty}}$ as a $Z$-module is not a $W$-fully visible module. Since, every submodule of $Z_{P^{\infty}}$ has the form $\left(1 / P^{l}+Z\right), l$ is a non-negative integer, then, there is no non-zero proper ideal $J$ of $Z$ such that $J\left(1 / P^{l}+Z\right)=\left(1 / P^{l}+Z\right)$. 
5) All modules of fully visible are $W$-fully visible, but the opposite is not true in general, and the following example explains that: $A$ module $Z_{4}$ on a ring $Z$ is a $W$-fully visible module by number (1), but it is not a fully visible module by (2).

6) A module $X$ over a field $T$ is not a $W$-fully visible, especially for $Q$ as a $Q$-module.

Proof. The result is directly from Remarks and Examples ((2.2), (14)).

7) Every simple T-module is W-fully visible

Proof. Suppose $W$ is a proper submodule of $X$, but $X$ is a simple module, then, $(0)$ and $X$ is the only submodule of $X$ and hence, the submodule $W$ must be zero (since $W$ is a proper submodule).

8) A proper submodule of $W$-fully visible module is also $W$-fully visible. Note that module $X$ over $T$ is not necessary to be a $W$-fully visible module when any submodule of it is $W$-fully visible. Consider the following example: the module $Z \oplus Z_{6}$ as a $Z$-module is not a $W$-fully visible module while the submodule $(0) \oplus Z_{6}$ of it is a $W$-fully visible module, since all proper submodules $(0) \oplus(\overline{0}),(0) \oplus(\overline{2}),(0) \oplus(\overline{3})$ are $W$-visible submodules of $(0) \oplus Z_{6}$. Therefore, $(0) \oplus Z_{6}$ is a $W$-fully visible $Z$-module.

Results can be obtained from (8), note next:

9) Let $X_{1}$ and $X_{2}$ be two $W$-fully visible $T$-modules; then, $X_{1} \cap X_{2}$ is also a $W$-fully visible module.

10) As a generalization of (9), let $\left(X_{i}\right\}_{i=1}^{n}$ be a family of $W$-fully visible $T$-modules; then, $\bigcap_{i=1}^{n} X_{i}$ is a $W$-fully visible module.

11) A direct summand of $W$-fully visible module $X$ is always $W$-fully visible.

Proposition 3.1. The homomorphic image of $W$-fully visible is also $W$-fully visible.

Proof. Let $f: \widehat{X} \longrightarrow \widehat{N}$ be an epimorphism where $\widehat{X}, \widehat{N}$ are two $T$-modules. Suppose $\widehat{X}$ is $W$-fully visible; let $\widehat{W}$ be a proper submodule of $\widehat{N}$. Then, $\widehat{W}=f(W)$ for some proper submodule $W$ of $\widehat{X}$, but $W$ is a $W$-visible submodule of $\widehat{X}$; then, there exists a non-zero proper ideal $C$ of $T$, implying that $W=C W$. Now, $\widehat{W}=f(W)=f(C W)=C f(W)=C \widehat{W}$.

Therefore, $\widehat{W}$ is a $W$-visible submodule of $\widehat{N}$; thus, $\widehat{N}$ is $W$-fully visible.

Proposition 3.2. Let $\widehat{X}_{1}$ and $\widehat{X}_{2}$ be two T-module; if $\widehat{X}_{1} \simeq \widehat{X}_{2}$, then, $\widehat{X}_{1}$ is a W-fully visible module if and only if $\widehat{X}_{2}$ is a $W$-fully visible module.

Proof. Suppose $\widehat{X}_{1}$ is a $W$-fully visible module; let $\widehat{W}$ be a proper submodule of $\widehat{X}_{2}$, and $T: \widehat{X}_{1} \longrightarrow \widehat{X}_{2}$ be isomorphism, then, there exist $W<\widehat{X}_{1}$ such that $T(W)=\widehat{W}$, but $\widehat{X}_{1}$ is $W$-fully visible module, then, $W$ is a $W$-visible submodule of $\widehat{X}_{1}$, that is, $W=I W$ for some ideal $I$ of $T$.

This implies that $\widehat{W}=T(W)=T(I W)=I T(W)=I \widehat{W}$; hence, $\widehat{W}$ is a $W$-fully visible module of $\widehat{X}_{2}$. Thus, $\widehat{X}_{2}$ is a $W$-fully visible module.

Opposite direction : let $W$ be a proper submodule of $\widehat{X}_{1}$; then, $T(W)$ is a proper submodule of $\widehat{X}_{2}$, implying the existence of a proper submodule $\widehat{W}$ of $\widehat{X}_{2}$ such that $T(W)=\widehat{W} \ldots(1)$.

But $\widehat{W}$ is a $W$-visible submodule (since $\widehat{X}_{2}$ is $W$-fully visible module); therefore, there exists an ideal $I$ of $T$, and implies that $\widehat{W}=I \widehat{W}$; hence, from (1), we get $T(W)=I T(W)$; this gives $T(W)=T(I W)$, but $T$ is monomorphism. Therefore, $W=I W$, leading to $W$ being a $W$-visible submodule and hence, $\widehat{X}_{1}$ is a $W$-fully visible module.

Proposition 3.3. Let $X$ be a $T$-module and $I$ is an ideal of $T$, such that $I \subseteq$ ann $_{T}(X)$; then, $X$ is a W-fully visible $T$-module if and only if $X$ is a $W$-fully visible $T / I$-module.

Proof. $\Longrightarrow)$ let $X$ be a $W$-fully visible $T$-module. Then, for every proper submodule $W$ of $X$, there exists a non-zero proper ideal $J$ of $T$ such that $W=J W$. Now, let $x \in W$, then, $x=\sum_{i=1}^{n} r_{i} a_{i}$ where $r_{i} \in J$ and $a_{i} \in W$, implying that $x=\sum_{i=1}^{n}\left(r_{i}+I\right) a_{i}$ where $r_{i}+I \in J / I, a_{i} \in W$.

Therefore, $x \in J / I W$, and $W \subseteq(J / I) W$. It is clear that $(J / I) W \subseteq W$ which implies that $W=(J / I) W$. Thus, $W$ is a $W$-visible $T / I$-submodule of $X$; this leads us to $X$ being a $W$-fully visible $T / I$-module of $X$.

$\Longleftarrow)$ Suppose $X$ is a $W$-fully visible $T / I$-module of $X$, then, for every proper submodule $W$ of $X$, there exists a nonzero proper ideal $J / I$ of $T / I$ such that $W=J / I W$. Now, let $m \in W$; then, $m=\sum_{i=1}^{n}\left(s_{i}+I\right) b_{i}$ where $\left(s_{i}+I\right) \in J / I$ and $b_{i} \in W$, which implies that $m=\sum_{i=1}^{n} s_{i} b_{i} \in J W$ where $s_{i} \in J$ and $b_{i} \in W$ and hence, $W \subseteq J W$ and we have $J W \subseteq W$; 
therefore, $J W=W$. This means that $W$ is a $W$-visible submodule of a $T$-module $X$ and hence, $X$ is a $W$-fully visible $T$-module.

Here, we will review the necessary and sufficient conditions for a module to be $W$-fully visible under the definition of tensor product.

Definition 3.2. : [3]

Let $X_{1}$ and $X_{2}$ be two $T$-modules; then, a tensor product of $X_{1}$ and $X_{2}$ is a pair $(X, F)$ where $X$ is a T-module and $F: X_{1} \times X_{2} \longrightarrow X$ is a bilinear map, such that for any $T$-module $N$ and any bilinear map $g: X_{1} \times X_{2} \longrightarrow N$, there exist a unique homomorphism $l: X \longrightarrow N$, such that $l o F=g$.

We demonstrate the following lemma to help us in giving our next major proposition.

Lemma 3.1. Let $X$ be a module over a ring $T$ and $N$ is a proper submodule of $X$. Then, $N \otimes T / I \simeq N / I N$ for any ideal $I$ of $T$.

Proof. Let $g: N \times T / I \longrightarrow N / I N$ such that $g(a, r+I)=r a+I N$

Where $a \in N$ and $r+I \in T / I$. It is easy to prove that $g$ is well defined and bilinear mapping. Now, to show that $g$ is an epimorphism; let $a+I N \in N / I N$, then, $g(a, 1+r)=a+I N$, where $(a, 1+r) \in N \otimes T / I$, that is, $g$ is an epimorphism and hence, there exits a homomorphism $l: N \otimes T / I \longrightarrow N / I N$ such that $l(a \otimes(r+I))=r a+I N$.

Where $T$ is a bilinear mapping. Then. by the first fundamental theorem $\frac{N \times T / I}{\operatorname{Kerl}} \simeq N / I N$;

$$
\begin{aligned}
\text { Kerl } & =\left(\sum_{i=1}^{n} a_{i} \otimes\left(r_{i}+I\right) \in N \otimes T / I ; l\left(\sum_{i=1}^{n} a_{i} \otimes\left(r_{i}+I\right)\right)=I N\right\} . \\
& =\left(\sum_{i=1}^{n} a_{i} \otimes\left(r_{i}+I\right) \in N \otimes T / I ; l\left(\sum_{i=1}^{n} a_{i} \otimes r_{i}(1+I)\right)=I N\right\} \\
& =\left(\sum_{i=1}^{n} a_{i} \otimes\left(r_{i}+I\right) \in N \otimes T / I ; l\left(\sum_{i=1}^{n} r_{i} a_{i} \otimes(1+I)\right)=I N\right\} \\
& =\left(\sum_{i=1}^{n} r_{i} a_{i}+I N=0=I N\right\} \text { hence } \sum_{i=1}^{n} r_{i} a_{i} \in I N .
\end{aligned}
$$
I)

Therefore, $\sum_{i=1}^{n} r_{i} a_{i}=\sum_{i=1}^{n} s_{j} b_{j}$ where $s_{j} \in I$ and $b_{j} \in N$. Therefore, $\sum_{i=1}^{n} a_{i} \otimes\left(r_{i}+I\right)=\sum_{i=1}^{n} r_{i} a_{i} \otimes(1+I)=\sum_{i=1}^{n} s_{j} b_{j} \otimes(1+$

$$
=\sum_{i=1}^{n} b_{j} \otimes\left(s_{j}+I\right)=\sum_{i=1}^{n} b_{j} \otimes 0=0
$$

Thus, $N \otimes T / I \simeq N / I N$.

The next theorem submits a characterization for a module to be a $W$-fully visible module under the tensor product.

Theorem 3.1. Let $X$ be a module over a ring $T$ and $N$ be a proper submodule of $X$. Then, $X$ is $W$-fully visible if and only if $\operatorname{ann}(N \otimes T / I)=T$

Proof. Assume that $X$ is a $W$-fully visible module and $N$ be a proper submodule of $X$; then, $N$ is a $W$-fully visible submodule of $X$. We have from Lemma (3.7), $N \otimes T / I \simeq N / I N$ for all ideal $I$ of $T$.

Hence, $\operatorname{ann}(N \otimes T / I)=\operatorname{ann}(N / I N)=(I N: N)=T$ by Proposition $((2.4),(2))$.

Another side: Suppose that $\operatorname{ann}(N \otimes T / I)=T$, we want to show that $X$ is a $W$-fully visible module. Therefore, we must prove that all proper submodules of $X$ are $W$-visible. Since $N$ is a proper submodule of $X$ and we have from Lemma (3..7), $N \otimes T / I \simeq N / I N$ for all ideal $I$ of $T$;

Therefore $T=\operatorname{ann}(N \otimes T / I)=\operatorname{ann}(N / I N)=(I N: N)$.

That is, $(I N: N)=T$ and by Proposition $((2.4),(2)), N$ is a $W$-visible submodule. Thus, $X$ is a $W$-fully visible module.

\section{REFERENCES}

[1] M. S. Fiadh and B. N. Shihab, "Visible Submodules of a Module X Over a Ring R is Introduced," Journal for Engineering and Applied Sciences, vol. 13, no. 24, pp. 10349-10355, 2018.

[2] M. S. Fiadh, "Visible (W-Visible) Submodules and Fully Visible (W-Fully visible) Modules With Some of Their Generalizations, Ph.D. Dissertation, university of Baghdad," 2019.

[3] P. B. Garrett, "Abstract Algebra," 2007. CRC Press (Taylor and Francis group). 